\title{
¿Diversidad o dominancia en la producción de alimentos? El caso de los polinizadores
}

\author{
Lucas A. Garibaldi ${ }^{1 \otimes}$; Sebastián Aguiar ${ }^{2}$; Marcelo A. Aizen ${ }^{3}$; Carolina L. \\ Morales ${ }^{3}$ \& Agustín SÁEZ \\ ${ }^{1}$ Instituto de Investigaciones en Recursos Naturales, Agroecología y Desarrollo Rural (IRNAD), Sede Andina, Universidad \\ Nacional de Río Negro (UNRN) - Consejo Nacional de Investigaciones Científicas y Técnicas (CONICET). San Carlos de \\ Bariloche, Río Negro, Argentina. ${ }^{2}$ Laboratorio de Análisis Regional y Teledetección. IFEVA, Universidad de Buenos Aires, \\ CONICET, Facultad de Agronomía, CABA. ${ }^{3}$ Laboratorio Ecotono, Instituto de Investigaciones en Biodiversidad y Medio \\ Ambiente (INIBIOMA), Centro Regional Universitario Bariloche, Universidad Nacional del Comahue - Consejo Nacional de \\ Investigaciones Científicas y Técnicas (CONICET). San Carlos de Bariloche, Río Negro, Argentina.
}

\begin{abstract}
Resumen. La biodiversidad está siendo destruida a una tasa alarmante. Una de las principales causas de esta pérdida es el cambio de uso del suelo, que se basa en la agricultura y la ganadería convencionales. Las prácticas de manejo como el monocultivo y el uso intensivo de agroquímicos reducen el número de especies de plantas, aves, insectos y otros grupos taxonómicos, a la vez que aumentan la abundancia relativa (dominancia) de pocas especies cultivadas y silvestres (e.g., malezas). Dado que casi $40 \%$ de la superficie terrestre se destina a la producción de cultivos y de carne, es clave lograr una producción agropecuaria compatible con la preservación de la biodiversidad. Además de su valor por aspectos éticos, espirituales y de uso para generaciones futuras, en este artículo destacamos el rol de la biodiversidad en la producción agropecuaria, y usamos a los polinizadores como ejemplo. Paradójicamente, la agricultura convencional está destruyendo la diversidad de polinizadores, pero esta diversidad es fundamental para incrementar la productividad (y su estabilidad en tiempo y espacio) de muchos cultivos. Varios estudios demuestran que la pérdida de diversidad de polinizadores no se puede compensar con una abundancia alta de una sola especie de polinizador (dominancia). Es por ello que debatimos acciones que pueden tomar los productores, consumidores, políticos y científicos para recuperar parte de esta biodiversidad. Por ejemplo, los productores pueden implementar prácticas dentro y fuera del cultivo para aumentar los recursos florales y de nidificación a los polinizadores y, de este modo, promover su abundancia y diversidad. Además, los consumidores pueden modificar su dieta, reducir los desperdicios y producir alimentos a pequeña escala, entre otras acciones. Como consecuencia, resulta imperioso tomar acciones múltiples por todos los actores, pues una sola estrategia no será suficiente para resolver el dilema de producir y conservar la biodiversidad.
\end{abstract}

[Palabras clave: agricultura, consumo, cultivos, degradación, equidad, intensificación, polinización, seguridad alimentaria, servicios ambientales, sustentabilidad]

Abstract. Diversity or dominance in food production? The case of pollinators. Biodiversity is being lost at an alarming rate. One of the main causes of this loss is the land-use change caused by the expansion of conventional agriculture and livestock production. Management practices such as monocultures and the intensive use of agrochemicals reduce the number of species of plants, birds, insects and other taxonomic groups, and increase, at the same time, the relative abundance (dominance) of one or a few cultivated and wild (e.g., weed) species. Given that $\sim 40 \%$ of the terrestrial surface is occupied by crop and livestock lands, it is critical to increase food production without destroying biodiversity. In addition to the value given by its ethical and spiritual dimensions, and the potential use of future generations, in this article we discuss the value of biodiversity for agriculture, using pollinators as a case of study. Paradoxically, conventional agriculture is reducing pollinator diversity, but this diversity is necessary for increasing productivity (and its temporal and spatial stability) of many crops. Several studies show that the loss of wild pollinator diversity cannot be replaced by a high abundance of a single pollinator species (dominance). Therefore, we discuss actions that producers, consumers, politicians and scientists can take to recover diversity. For example, producers can implement management practices in- and outside the crop fields to increase floral and nesting resources, and therefore pollinator abundance and diversity. In addition, consumers can modify diets, reduce waste and produce food at small scales, among many other actions. One single strategy will not be enough to solve the dilemma of producing food and preserving biodiversity. We argue that multiple actions must be taken urgently from all the stakeholders.

[Keywords: agriculture, consumption, crop, degradation, evenness, intensification, pollination, food security, ecosystem services, sustainability]

Editor asociado: Esteban Jobbágy 


\section{DOMINANCIA EN REEMPLAZO DE DIVERSIDAD}

El problema es grave. Los seres humanos estamos destruyendo la biodiversidad e incrementando la dominancia (Cardinale et al. 2012). El manejo que implica la agricultura convencional tiene como resultado el reemplazo de muchas especies silvestres y cultivadas por una sola (e.g., la soja, el girasol o el maíz). La dominancia ocurre cuando una especie, ya sea cultivada o silvestre, se vuelve muy abundante en relación al resto. Los monocultivos representan una de las expresiones más altas de la dominancia. En ellos, una sola especie monopoliza la mayor parte de los recursos como el espacio, la luz, el agua y los nutrientes a lo largo de grandes extensiones, y deja poco y nada para el resto. En cambio, la coexistencia de muchas especies con abundancias relativamente similares, que representa la máxima expresión de la biodiversidad, es el resultado de una repartición relativamente equitativa de estos recursos. De hecho, la biodiversidad se retroalimenta, lo que fomenta más biodiversidad, ya que distintas especies (e.g., de plantas) representan hábitat y alimento para otras especies (e.g., de polinizadores).

Una de las principales causas de destrucción de la biodiversidad a nivel mundial es el cambio en el uso del suelo (Maxwell et al. 2016; Newbold 2016). Estos cambios están asociados a la producción agropecuaria necesaria para satisfacer una demanda creciente de alimentos y otros productos (azúcar, algodón, biocombustibles, etc.) de alto valor de mercado. Muchas veces pensamos nuestro planeta como un lugar lleno de selvas y maravillas naturales, y sin dudas que las hay, pero hoy en día, $40 \%$ de la superficie terrestre se destina a cultivos y a la producción animal. Si consideramos que gran parte del resto son desiertos y zonas urbanas, y que la mayor parte de las tierras más fértiles ya están bajo cultivo (Foley et al. 2011), podemos concluir que convertimos a nuestro planeta en una enorme granja. Como consecuencia de esta conversión estamos perdiendo una buena parte de nuestro acervo biológico.

Pero ¿por qué debemos preocuparnos en mantener y fomentar la diversidad? La respuesta más inmediata es que la biodiversidad representa una parte importante de nuestro legado como especie, de la cual hemos seleccionado aquellos organismos que alimentan nuestros estómagos y espíritus, y que debe ser preservada para las generaciones futuras. Más aun, en las últimas décadas existe una conciencia creciente de que la biodiversidad está conectada con la productividad (Cardinale et al. 2012), por lo que adquiere una nueva dimensión que tiene implicancias directas en aspectos tan tangibles como son la salud humana y la economía. Es clave, entonces, producir alimentos sin seguir destruyendo la biodiversidad.

En este artículo discutimos la importancia de la diversidad para la producción agrícola y usamos como ejemplo nuestra experiencia de trabajo con los sistemas planta-polinizador. Luego debatimos algunas acciones que pueden realizar los productores, consumidores, políticos y científicos para promover la biodiversidad (o para, al menos, disminuir su tasa de destrucción) en los paisajes agrícolas. Argumentamos que se necesitan múltiples acciones por parte de todos los actores, ya que una sola estrategia aislada no será suficiente.

\section{¿Cuáles son las consecuencias DE REEMPLAZAR DIVERSIDAD POR DOMINANCIA? EL CASO DE LOS POLINIZADORES}

Frente a la pérdida creciente de biodiversidad y de los hábitats naturales que la alberga, la aparente solución consiste en reemplazar diversidad por dominancia. En el caso de la polinización animal, ello contrapone dos paradigmas. Uno, que mayor productividad agrícola se alcanza al aumentar el número de, por ejemplo, abejas, más allá de qué especie sea (dominancia). El otro, donde este aumento en la productividad sólo se alcanza si el incremento en el número de abejas se logra a través de un mayor número de especies (diversidad). Estos dos paradigmas fueron evaluados en más de 600 campos en cinco continentes, en un trabajo coordinado con más de 50 científicos durante varios años (Garibaldi et al. 2013). Más arriba mencionamos cómo el monocultivo (dominante) reemplaza la diversidad de plantas. En nuestros estudios evaluamos las consecuencias de este reemplazo, pero considerando a los polinizadores y la polinización de cultivos. Cada vez que cerramos los ojos y pensamos en un polinizador, se nos viene a la mente la abeja melífera (Apis mellifera). Es la abeja que criamos en colmenas y nos da la tan preciada miel, entre muchos otros beneficios. La abeja melífera es la más abundante y dominante a nivel global, visita flores de 
cultivos transportando polen de una flor a otra y favorece la formación de los frutos y semillas que luego consumimos, volvemos a sembrar o usamos para alimentar al ganado. Sin embargo, la abeja melífera es sólo una de las más de 20 mil especies de abejas que hay en el mundo, sin contar los cientos o miles de especies de mariposas, moscas, escarabajos, aves y murciélagos, entre otros, que también son polinizadores. Nuestros resultados mostraron cuatro escenarios en términos de abundancia y diversidad de polinizadores, que se reflejan en un gradiente de menor a mayor cuajado de frutos en los campos de cultivo: a) baja abundancia de abeja melífera y de polinizadores silvestres, $b$ ) alta abundancia de abeja melífera, c) alta abundancia de una fauna diversa de polinizadores silvestres, y d) alta abundancia de abeja melífera y de polinizadores silvestres (Garibaldi et al. 2013). Encontramos que la diversidad no puede ser reemplazada por una alta abundancia de un sólo tipo de polinizador (dominancia). Incrementar la diversidad aumenta el cuajado de frutos sin importar la cantidad de colmenas de la abeja melífera.

En general, mayor diversidad de especies implica mayor cantidad de funciones ecosistémicas, así como una mayor estabilidad de estas funciones en el espacio y en el tiempo (Tilman et al. 2014). Estas ideas, por lo general analizadas en relación a la dinámica del carbono y nutrientes (productividad primaria neta, descomposición, etc.) (Lefcheck et al. 2015), fueron recientemente analizadas para la polinización. Se demostró que la diversidad de polinizadores es clave por múltiples razones (Garibaldi et al. 2015). Por ejemplo, distintos polinizadores polinizan distintos tipos de flores. Cultivos con flores grandes son polinizados por visitantes florales de mayor tamaño que pueden contactar sus estructuras reproductivas, mientras que cultivos con flores tubulares son mejor polinizados por visitantes de lengua larga. Además, el clima es intrínsecamente variable y está cambiando, por lo que tener diversidad de polinizadores garantiza la polinización ante distintas condiciones climáticas. Hay días que hace frío y sólo algunas especies de polinizadores pueden volar en estas circunstancias, mientras que los días de más calor están más activas otras especies. Además, distintas especies de polinizadores pueden variar en cuanto a su patrón de actividad diaria y comportamiento de forrajeo. Por ejemplo, especies de polinizadores actúan en distintas flores de los cultivos según su posición y orientación, lo que garantiza la polinización de las flores presentes en toda la planta. En resumen, hay múltiples evidencias de que distintas especies se complementan y actúan de manera sinérgica para producir más alimentos, tanto en cantidad como en calidad y variedad (Brittain et al. 2013). Esto implica que es necesaria una diversidad de polinizadores para proveer una adecuada polinización a una diversidad de cultivos. Incluso, para polinizar un solo cultivo es beneficioso contar con muchas especies de polinizadores.

\section{¿CuÁles SON LAS TENDENCIAS?}

Las tendencias son alarmantes. Seguiremos destruyendo la biodiversidad. Sin embargo, tendremos casi que duplicar la producción agrícola en los próximos 40 años (Foley et al. 2011). Una mayor expansión agropecuaria (monocultivo) está asociada a menor diversidad de plantas, lo que implica menor diversidad de polinizadores; esto redunda en menor cantidad y calidad de polen en las flores de los cultivos y, por lo tanto, menor cantidad de semillas o frutos cosechadas por hectárea (rendimiento) en cultivos dependientes de polinizadores (Garibaldi et al. 2014). Si se produce menos por unidad de superficie, se expande aun más la superficie para satisfacer las demandas (Figura 1). A su vez, una mayor superficie agropecuaria implica menor diversidad de plantas. Esto resulta en un ciclo que se retroalimenta, lo que genera un círculo vicioso (Figura 1). Resulta clave, entonces, transformar este ciclo negativo en uno positivo, o círculo virtuoso. Hay muchos actores que pueden realizar múltiples acciones para transformar esta realidad. A continuación, planteamos un conjunto de recomendaciones dirigidas a cuatro actores esenciales en la preservación de la biodiversidad en los agroecosistemas: productores, consumidores, políticos y científicos.

\section{¿QUÉ PUEDEN HACER LOS PRODUCTORES?}

Lo habitual en el mundo es que la intensificación convencional, basada en el monocultivo y un gran uso de agroquímicos, implique más producción a costa de menor biodiversidad. Sin embargo, se puede transformar este ciclo destructivo en constructivo si se trae la biodiversidad nuevamente a los campos (Garibaldi et al. 2014). Por ejemplo, en los bordes de los cultivos, sin competir con el área cultivada, se puede 


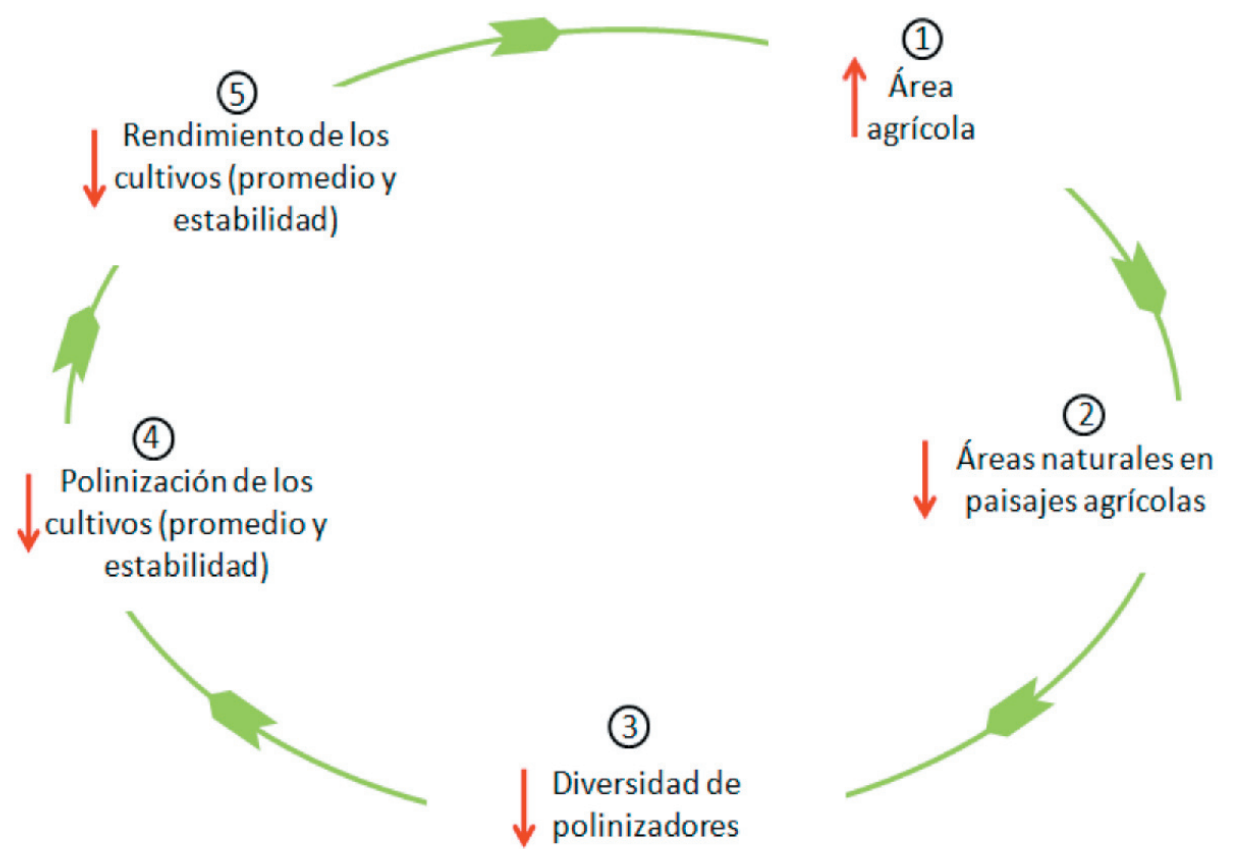

Figura 1. Ciclo de la declinación de la diversidad de polinizadores en paisajes agrícolas (modificado a partir de Garibaldi et al. [2014]). Si bien focalizamos nuestra discusión en la relación causal representada por este ciclo, reconocemos que cada variable depende también de múltiples otros factores. Ver texto principal para más información sobre los números.

Figure 1. Declination cicle in pollinator diversity within agricultural landscapes (modified from Garibaldi et al. [2014]). Although we focus our discussion in the causal relation represented by this cicle, we recognise that many other factors affect each variable. See main text for further detail about the numbers.

promover la diversidad de especies herbáceas, arbustivas y arbóreas que brinden hábitat para polinizadores, al proveer recursos florales y de anidamiento (Figura 1, etapa 2). Desde estos bordes, los polinizadores visitan los cultivos aledaños y aumentan su rendimiento. Para que esta práctica sea eficiente es importante que las parcelas cultivadas no presenten grandes extensiones, ya que al incrementarse la distancia desde estos bordes de cultivo que actúan como "fuente" de polinizadores decrece la abundancia y diversidad de los mismos. Además, la conservación y expansión de los fragmentos naturales y semi-naturales dentro de paisajes agrícolas también brinda hábitat para polinizadores y provee polinización a los cultivos (Garibaldi et al. 2011). Los hábitats amigables con los polinizadores son amigables para el ser humano.

¿Cuánto podemos aumentar el rendimiento si promovemos la diversidad de polinizadores? Históricamente se ha reconocido en la producción agrícola la importancia de la luz, el agua, los nutrientes y el control plagas y malezas para aumentar el rendimiento de los cultivos. Sin embargo, se le prestó mucha menos atención a un factor con gran incidencia: una polinización adecuada. La magnitud del efecto es clave, ya que de ello depende la relevancia agronómica de la diversidad. En un estudio a nivel global encontramos que en muchos cultivos (e.g., frambuesa, girasol, manzana, nabo, pepino, poroto, tomate y trigo sarraceno) el rendimiento puede aumentar un $25 \%$ en campos con ensambles de polinizadores más diversos, comparados con ensambles más pobres (Garibaldi et al. 2016). En conclusión, la promoción de la diversidad de polinizadores puede convertirse en una herramienta concreta de manejo en los campos de cultivo, con efectos importantes y medibles en el rendimiento de muchas variedades (Figura 1, etapas 3, 4 y 5). Esto es fundamental debido a que cada vez sembramos una proporción mayor de cultivos dependientes de polinizadores en relación a los no dependientes (Aizen et al. 2008).

\section{¿Qué PUEDEN HACER LOS CONSUMIDORES?}

Las predicciones respecto duplicar la producción en los próximos 40 años se basan en nuestras tendencias de consumo. Cada vez somos más, pero el aspecto clave es que el consumo per cápita aumenta. Si bien existen enormes asimetrías entre países, regiones, y estratos socioeconómicos en el acceso a los alimentos, en promedio, a nivel global, 
nuestros hijos consumen más que nosotros; a su vez, nosotros consumimos más que nuestros padres, y así sucesivamente. Como consumidores, muchos vivimos en ciudades y estamos lejos del campo, por lo que pensamos que no somos responsables de los cambios en el uso del suelo. Sin embargo, nuestros patrones de consumo ejercen una gran influencia y su modificación podría generar una reducción en la cantidad de tierra necesaria para alimentar a la humanidad (Figura 1, etapa 1).

¿Realmente se necesita producir más y seguir destruyendo la biodiversidad? ¿O podemos cambiar nuestros hábitos de consumo? Cuando pensamos en aumentar la producción agrícola lo asociamos a satisfacer las necesidades de alimentos de la población desnutrida. Sin embargo, hoy en día hay alimento suficiente para toda la población del mundo, aunque una parte importante de ella no pueda acceder al mismo. Si bien $\sim 10 \%$ de la población mundial sufre de desnutrición, $40 \%$ tiene sobrepeso (y en la Argentina, la incidencia del sobrepeso es mayor a 60\%) (World Health Organization 2016). En consecuencia, la principal limitante actual para reducir la malnutrición y garantizar la seguridad alimentaria no parecen ser los niveles de producción agrícola global, sino más bien aspectos vinculados a la inequidad en el acceso y la utilización de alimentos saludables, y a factores culturales heredados, entre ellos los hábitos de consumo por la falta de educación y de auto-conocimiento.

Hay varias maneras de reducir la necesidad de más producción agrícola para abastecer a esta demanda creciente, modificando nuestros patrones de consumo. En primer lugar, es importante que reduzcamos los desperdicios (Foley et al. 2011). Un tercio de la comida se descarta en los distintos tramos de la cadena que va desde la producción hasta el consumo. Tenemos que estar pensando constantemente en cómo reducir los desperdicios en nuestra vida cotidiana. Por ejemplo, habitualmente en verdulerías, supermercados y restaurantes se tiran a la basura verduras y frutas que son nutricionalmente aptas pero que tienen pequeños "defectos" visuales o de forma que hacen que los consumidores no las elijamos. También, el sistema de producción es ineficiente debido a la distancia que existe en muchos casos entre los mercados productores y consumidores, así como a las oscilaciones en los precios de estos "commodities". En este sentido, es interesante potenciar el mercado sin intermediación donde el contacto entre el productor y el consumidor permite que se sostenga la producción no masiva y la calidad, con potenciales beneficios económicos para ambas partes.

Otro aspecto importante es que, a grandes rasgos, no deberíamos consumir más de 300 g de carne de mamíferos o aves por persona y por semana. Según estudios académicos ello tendría muchos beneficios para nuestra salud. Además, tiene beneficios para el planeta. Casi la mitad de la producción agrícola se destina a alimentar animales (Foley et al. 2011). Ello es muy ineficiente, dado que, por ejemplo, sólo 3\% de la energía del maíz llega a los humanos a través del consumo de carne de vaca que es alimentada con maíz, comparado con el consumo directo del maíz. Si comiésemos menos carne, necesitaríamos menos hectáreas de cultivo en el mundo para obtener la misma cantidad de calorías. Es importante resaltar que cualquier reducción que hagamos en el consumo de carne que fue producida en base a granos es beneficiosa para nuestra salud y el planeta, aunque sea pequeña.

Si bien hay muchos cambios que podemos realizar los consumidores, el último aspecto que queremos discutir aquí es que los consumidores podemos transformarnos en productores. A escala individual o familiar podemos cultivar alimentos en macetas dentro de departamentos y casas, así como en nuestros jardines. Muchos de nosotros somos afortunados en tener jardines y los elegimos porque nos gusta la naturaleza. Sin embargo, el manejo habitual de esos jardines es reemplazar la diversidad de flora nativa por una sola especie de pasto, al cual le proveemos muchísima agua potable. Nos gusta ver nuestros jardines homogéneos y limpios, de la misma forma como nos gusta ver nuestros campos de cultivo y la verdura y fruta que consumimos. Lo aconsejable es hacer lo mismo que le pedimos a los productores, traer la diversidad de nuevo a nuestros jardines. Por supuesto de las plantas nativas que habitaban allí, pero también de frutales y vegetales. En una escala de vecindario, también podemos promover las huertas comunitarias en sitios como parques, escuelas, o baldíos, así como utilizar frutales para el arbolado urbano, de calles y espacios públicos. Más allá de reducir la demanda de alimentos por producirlos en nuestros domicilios, pensamos que este proceso es revelador. Plantar una semilla, regarla, cuidarla, y ver que se transforma en una planta, de la cual podemos consumir, por ejemplo, tomates con olor y sabor a tomate nos cambia nuestra forma de percibir el 
mundo, de pensar, de actuar, de valorar la comida, y nos ayuda a comprender mejor el sistema agroalimentario. También contribuye a valorar más el trabajo humano por detrás de la producción de alimento a pequeña escala o de agricultura familiar, generando una elección más responsable de los alimentos que adquirimos.

\section{¿Qué PUEDEN HACER LOS POLÍTICOS?}

Históricamente, la estrategia comúnmente empleada para la conservación de la biodiversidad y hábitats ha sido la creación de áreas protegidas públicas (Figura 1, etapa 2). Sin embargo, los campos productivos suelen ser tierras privadas donde las decisiones de manejo las toma el propietario. Por ello, en distintas partes del mundo se han empleado políticas que buscan modificar los usos de la tierra y las prácticas de manejo que realizan los productores, a través de regulaciones gubernamentales e incentivos económicos. Por lo general, estos últimos se basan en acuerdos voluntarios con los productores, a quienes se los compensa por medio de diferentes instrumentos económicos, por la externalidad positiva que proveen al conservar la biodiversidad. Lamentablemente, en la Argentina, las leyes o incentivos que promuevan la preservación de hábitats claves en el paisaje agrícola son aún muy incipientes. Tenemos un largo camino por delante de aprendizaje para lograr prácticas de manejo ambientalmente amigables y eficientes, con el gobierno como actor principal.

Un aspecto en el que la intervención gubernamental es clave para garantizar la diversidad de polinizadores silvestres y la sanidad no sólo de estos, sino también de las abejas de la miel, es en la regulación del comercio de polinizadores producidos en forma masiva (Figura 1, etapa 3). Entre estos se incluyen la misma abeja de la miel, pero también a las colonias comerciales de abejorros (Bombus spp.), con frecuencia usados en la polinización de cultivos de invernadero. Por ejemplo, los abejorros $B$. terrestris utilizados y comercializados en todo el mundo son muy invasores, no sólo en los países donde son introducidos, sino también en los países vecinos, por lo que es imprescindible un abordaje supranacional o bilateral en estas decisiones. Asimismo, es importante conocer en detalle el comportamiento de estos polinizadores, ya que se puede generar un efecto no deseado y afectar de forma negativa la producción (Aizen et al. 2014).
La pérdida de biodiversidad, como tantos otros problemas ambientales, puede enmarcarse dentro de lo que se conocen como problemas de acción colectiva. En términos concretos, estos problemas surgen cuando los individuos actúan de forma egoísta, acaparan los beneficios económicos y socializan los costos ambientales; es decir, perjudican el bienestar colectivo. En el contexto de la polinización y la biodiversidad, las soluciones a estos problemas se pueden dar a través de incentivos económicos y mediante regulaciones gubernamentales, como se indicó en párrafos anteriores. Sin embargo, también es posible, y sobre todo necesario, que los individuos actúen de forma colectiva para afrontar estos problemas a través de la modificación de normas o conductas sociales, y no sólo mediante regulaciones o incentivos (Nyborg et al. 2016). Creemos que para modificar estas conductas, un primer paso indispensable es que los políticos hagan un esfuerzo por promover la educación respecto a cómo nuestras dietas y hábitos no sólo modifican nuestra salud, sino también la del medio ambiente.

\section{¿Qué PUEDEN HACER LOS CIENTÍFICOS?}

La participación de la ciencia y los científicos es clave cuando se debe disminuir la incertidumbre asociada a la toma de decisiones (Paruelo 2016). Lamentablemente, la función que cumplen los científicos como generadores de conocimientos no está debidamente validada e internalizada, y tampoco está dimensionado e instrumentado cómo estos conocimientos se usan para modificar la realidad. Hoy no es frecuente que los tomadores de decisiones vengan a nuestras oficinas a golpear la puerta para preguntar si estamos de acuerdo respecto de decisiones o políticas sobre el uso de la tierra, del aire y del agua. Tenemos que salir y vincularnos en forma activa con los políticos, consumidores y productores, entre muchos actores (Fernández 2014). En este sentido, muchos investigadores participamos en plataformas intergubernamentales donde consensuamos la evidencia actual respecto los temas ambientales para tomar mejores decisiones políticas y cambiar legislación. Un ejemplo de ello es la Plataforma Intergubernamental para la Biodiversidad y los Servicios Ambientales (IPBES por sus siglas en inglés). De estas instancias de consenso surge como conclusión que la biodiversidad en general, y la de 
polinizadores en particular, es importante para nuestra supervivencia (Potts et al. 2016). Esta última no sólo aumenta la producción agrícola, sino también nos brinda beneficios múltiples. Por ejemplo, potencia actividades económicas como el turismo o la apicultura, es importante para la protección de fuentes de agua y la producción de oxígeno y es clave desde el punto de vista espiritual y ético.

En conclusión, los datos son claros: estamos destruyendo la biodiversidad y la estamos reemplazando por dominancia. Esto es un error, porque la biodiversidad es importante para nuestra supervivencia. Parte de esta diversidad se puede recuperar, pero tenemos que actuar rápido. ¿Biodiversidad o dominancia en la producción de alimentos? ¡Biodiversidad!

Agradecimientos. Agradecemos el financiamiento de la Universidad Nacional de Río Negro en investigación (PI 40-B-399) y extensión (Resolución Rectoral 315/16), así como de la Agencia Nacional de Promoción Científica y Tecnológica (FONCYT, PICT 2012-3015, PICT 2013-1079 y PICT 2015-2333). Georg Andersson, Roberto Fernández, Nancy García, Matías Goldenberg y Gonzalo Irisarri nos brindaron correcciones sobre versiones preliminares del manuscrito e ideas muy interesantes.

\section{REFERENCIAS}

Aizen, M. A., L. A. Garibaldi, S. A. Cunningham, and A. M. Klein. 2008. Long-term global trends in crop yield and production reveal no current pollination shortage but increasing pollinator dependency. Current Biology 18:15725 .

Aizen, M. A., C. L. Morales, D. P. Vázquez, L. A. Garibaldi, A. Sáez, and L. D. Harder. 2014. When mutualism goes bad: density-dependent impacts of introduced bees on plant reproduction. New Phytologist 204:322-328.

Brittain, C., N. Williams, C. Kremen, and A. M. Klein. 2013. Synergistic effects of non-Apis bees and honey bees for pollination services. Proceedings of the Royal Society B: Biological Sciences 280:20122767.

Cardinale, B. J., J. E. Duffy, A. Gonzalez, D. U. Hooper, C. Perrings, P. Venail, A. Narwani, G. M. Mace, D. Tilman, D. A. Wardle, A. P. Kinzig, G. C. Daily, M. Loreau, J. B. Grace, A. Larigauderie, D. S. Srivastava, and S. Naeem. 2012. Biodiversity loss and its impact on humanity. Nature 486:59-67.

Fernández, R. J. 2014. Decálogo del ambientalismo estéril. Ecología Austral 24:356-364.

Foley, J. A., N. Ramankutty, K. A. Brauman, E. S. Cassidy, J. S. Gerber, M. Johnston, N. D. Mueller, C. O'Connell, D. K. Ray, P. C. West, C. Balzer, E. M. Bennett, S. R. Carpenter, J. Hill, C. Monfreda, S. Polasky, J. Rockström, J. Sheehan, S. Siebert, D. Tilman, and D. P. M. Zaks. 2011. Solutions for a cultivated planet. Nature 478:337-342.

Garibaldi, L. A., I. Bartomeus, R. Bommarco, A. M. Klein, S. A. Cunningham, M. A. Aizen, V. Boreux, M. P. D. Garratt, L. G. Carvalheiro, C. Kremen, C. L. Morales, C. Schüepp, N. P. Chacoff, B. M. Freitas, V. Gagic, A. Holzschuh, B. K. Klatt, K. M. Krewenka, S. Krishnan, M. M. Mayfield, I. Motzke, M. Otieno, J. Petersen, S. G. Potts, T. H. Ricketts, M. Rundlöf, A. Sciligo, P. A. Sinu, I. Steffan-Dewenter, H. Taki, T. Tscharntke, C. H. Vergara, B. F. Viana, and M. Woyciechowski. 2015. Trait matching of flower visitors and crops predicts fruit set better than trait diversity. Journal of Applied Ecology 52:1436-1444.

Garibaldi, L. A., L. G. Carvalheiro, S. D. Leonhardt, M. A. Aizen, B. R. Blaauw, R. Isaacs, M. Kuhlmann, D. Kleijn, A. M. Klein, C. Kremen, L. Morandin, J. Scheper, and R. Winfree. 2014. From research to action: enhancing crop yield through wild pollinators. Frontiers in Ecology and the Environment 12:439-447.

Garibaldi, L. A., L. G. Carvalheiro, B. E. Vaissiere, B. Gemmill-Herren, J. Hipolito, B. M. Freitas, H. T. Ngo, N. Azzu, A. Saez, J. Astrom, J. An, B. Blochtein, D. Buchori, F. J. C. Garcia, F. Oliveira da Silva, K. Devkota, M. d. F. Ribeiro, L. Freitas, M. C. Gaglianone, M. Goss, M. Irshad, M. Kasina, A. J. S. P. Filho, L. H. P. Kiill, P. Kwapong, G. N. Parra, C. Pires, V. Pires, R. S. Rawal, A. Rizali, A. M. Saraiva, R. Veldtman, B. F. Viana, S. Witter, and H. Zhang. 2016. Mutually beneficial pollinator diversity and crop yield outcomes in small and large farms. Science (New York, N.Y.) 351:388-391.

Garibaldi, L. A., I. Steffan-Dewenter, C. Kremen, J. M. Morales, R. Bommarco, S. A. Cunningham, L. G. Carvalheiro, N. P. Chacoff, J. H. Dudenhöffer, S. S. Greenleaf, A. Holzschuh, R. Isaacs, K. Krewenka, Y. Mandelik, M. M. Mayfield, L. A. Morandin, S. G. Potts, T. H. Ricketts, H. Szentgyörgyi, B. F. Viana, C. Westphal, R. Winfree, and A. M. Klein. 2011. Stability of pollination services decreases with isolation from natural areas despite honey bee visits. Ecology Letters 14:1062-1072.

Garibaldi, L. A., I. Steffan-Dewenter, R. Winfree, M. A. Aizen, R. Bommarco, S. A. Cunningham, C. Kremen, L. G. Carvalheiro, L. D. Harder, O. Afik, I. Bartomeus, F. Benjamin, V. Boreux, D. Cariveau, N. P. Chacoff, J. H. Dudenhöffer, B. M. Freitas, J. Ghazoul, S. Greenleaf, J. Hipólito, A. Holzschuh, B. Howlett, R. Isaacs, S. K. Javorek, C. M. Kennedy, K. Krewenka, S. Krishnan, Y. Mandelik, M. M. Mayfield, I. Motzke, T. Munyuli, B. A. Nault, M. Otieno, J. Petersen, G. Pisanty, S. G. Potts, R. Rader, T. H. Ricketts, M. Rundlöf, C. L. Seymour, C. Schüepp, H. Szentgyörgyi, H. Taki, T. Tscharntke, C. H. Vergara, B. F. Viana, T. C. Wanger, C. Westphal, N. Williams, and A. M. Klein. 2013. Wild pollinators enhance fruit set of crops regardless of honey bee abundance. Science (New York, N.Y.) 339:1608-1611.

Lefcheck, J. S., J. E. K. Byrnes, F. Isbell, L. Gamfeldt, J. N. Griffin, N. Eisenhauer, M. J. S. Hensel, A. Hector, B. J. Cardinale, and J. E. Duffy. 2015. Biodiversity enhances ecosystem multifunctionality across trophic levels and habitats. Nature Communications 6:6936.

Maxwell, S. L., R. A. Fuller, T. M. Brooks, and J. E. M. Watson. 2016. The ravages of guns, nets and bulldozers. Nature 
536:146-145.

Newbold, T. 2016. Has land use pushed terrestrial biodiversity beyond the planetary boundary? A global assessment. Science (New York, N.Y.) 353:288-291.

Nyborg, K., J. M. Anderies, A. Dannenberg, T. Lindahl, C. Schill, M. Schüter, W. N. Adger, K. J. Arrow, S. Barrett, S. Carpenter, F. S. Chapin III, A. S. Crépin, G. Daily, P. Ehrlich, C. Folke, W. Jager, N. Kautsky, S. A. Levin, O. J. Madsen, S. Polasky, M. Scheffer, B. Walker, E. U. Weber, J. Wilen, A. Xepapadeas, and A. de Zeeuw. 2016. Social norms as solutions. Science 354:42-43.

Paruelo, J. M. 2016. El papel de la Ciencia en el proceso de Ordenamiento Territorial (y en otras cuestiones vinculadas con problemas ambientales ). Ecología Austral 26(1):51-58.

Potts, S. G., V. L. Imperatriz-Fonseca, H. T. Ngo, J. C. Biesmeijer, T. D. Breeze, L. V. Dicks, L. A. Garibaldi, R. Hill, J. Settele, A. J. Vanbergen, M. A. Aizen, S. A. Cunningham, C. Eardley, B. M. Freitas, N. Gallai, P. G. Kevan, A. KovácsHostyánszki, P. K. Kwapong, X. L. J. Li, D. J. Martins, G. Nates-Parra, J. S. Pettis, R. Rader, and B. F. Viana. 2016. IPBES (2016): Summary for policymakers of the assessment report of the Intergovernmental Science-Policy Platform on Biodiversity and Ecosystem Services on pollinators, pollination and food production.

Tilman, D., F. Isbell, and J. M. Cowles. 2014. Biodiversity and ecosystem functioning. Annual Review of Ecology, Evolution, and Systematics 45:471-493.

World Health Organization. 2016. Obesity and overweight. URL: www.who.int/mediacentre/factsheets/fs311/ en/ 